\title{
Corpus Callosum Impingement Syndrome: A Callosal or Colossal Problem?
}

\author{
Sheng-Fei Oon, Hong Kuan Kok, Christen D. Barras, Seamus Looby, \\ Paul Brennan, Hamed Asadi
}

Keywords: Magnetic resonance imaging, Neuroimaging

A 59-year-old female with a past history of advanced breast cancer with bone metastases presented with progressive headaches, nausea, visual disturbance on upward gaze, imbalance, and gait abnormalities. Magnetic resonance imaging (MRI) of the brain demonstrated an enhancing tectal mass extending into the periaqueductal grey matter causing severe obstructive hydrocephalus and anterior cerebellar leptomeningeal enhancement (Figure 1). The patient was treated with ventriculoperitoneal (VP) shunting, and the patient's symptoms improved following ventricular decompression; however, an upward gaze palsy persisted. Routine serology was normal, and an examination of the cerebrospinal fluid showed normal biochemistry and cell count. No organisms were cultured. In view of the clinical history, the mass was presumed to be a breast metastasis causing aqueduct stenosis and Parinaud's syndrome. A repeat MRI demonstrated decompression of the ventricular system with the VP shunt, but there was new extensive abnormal T2weighted and FLAIR hyperintensity throughout the corpus callosum and pericallosal white matter (Figure 2), with no corresponding enhancement (Figure 3C) or abnormal diffusion restriction (Figure 3D). A diagnosis of corpus callosum impingement syndrome (CCIS) was made. A further MRI in preparation for wholebrain radiotherapy 4 weeks later demonstrated complete resolution of the T2-weighted corpus callosum changes (Figure 4).

CCIS is an uncommon sequela of severe and usually chronic hydrocephalus, where there is stretching of the lateral ventricles ${ }^{1,2}$ and/or impingement of the corpus callosum against the undersurface of the cerebral falx. ${ }^{3}$ The rigid inferior margin of the falx impinges on the cranially expanding corpus callosum, resulting in flattening and thinning of the posterior body. ${ }^{4}$ The imaging features are characterised by T1-weighted signal hypointensity as well as T2-weighted and FLAIR signal hyperintensity within the corpus callosum, primarily involving its body and isthmus., There may be indentation of the cingulate gyrus, resulting in cerebrospinal fluid accumulation in the indented spaces. ${ }^{1}$ Longstanding cases may eventually demonstrate localized atrophic callosal volume loss and signal abnormality ${ }^{4,6}$ that may persist after the ventricular system has been decompressed. ${ }^{1,3,6}$

It is generally accepted that longstanding compression of the distended corpus callosum against the inferior surface of the falx is the likely cause for the imaging appearances. ${ }^{3}$ Various proposed mechanisms include oedema, corpus callosal softening from chronic hydrocephalus, forced ventricular drainage, chronic elevated intraventricular pressures, compromised venous drainage, diminished arterial supply, traction-induced arterial compromise, and demyelination. ${ }^{1-4}$

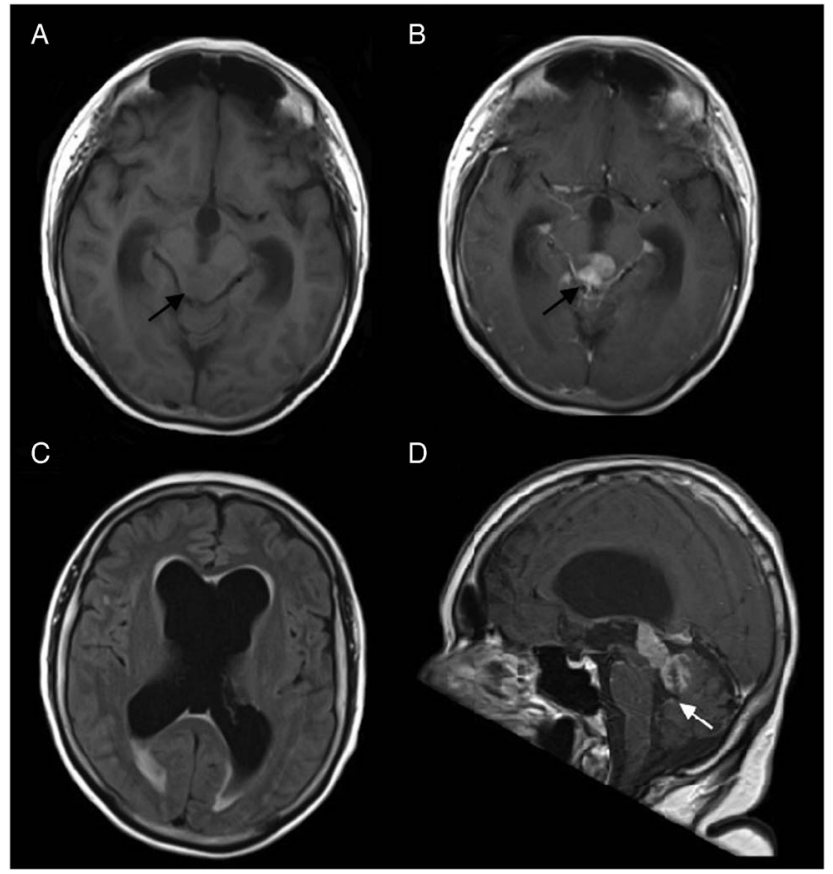

Figure 1: Axial pre- (A) and post-gadolinium (B) sequences demonstrating an enhancing mass in the midbrain tectum (black arrow). The mass extends to involve the pineal gland and surrounds the Sylvian aqueduct causing stenosis. There is severe hydrocephalus with transependymal oedema $(C)$ and cerebellar leptomeningeal enhancement (D). Note that the fourth ventricle is not enlarged (white arrow).

Ventricular decompression also leads to reversal of callosal signal change in most, but not all, cases of CCIS. ${ }^{1,4}$ In one case series, $75 \%$ of patients with follow-up MRI demonstrated

From the Department of Radiology, Neuroradiology Service, Beaumont Hospital, Dublin, Ireland (SFO, HKK, SL, PB); the Department of Radiology, St. Vincent's University Hospital, Dublin, Ireland (SFO); the Department of Interventional Radiology, Guy's and St. Thomas' NHS Foundation Trust, London, England (HKK); the Lysholm Department of Neuroradiology, The National Hospital for Neurology and Neurosurgery, Queen Square, London, England (CDB); the Department of Radiology, Neurointerventional Service, Austin Health, Melbourne, Australia (HA); the Neurointerventional Service, Monash Imaging, Monash Health, Melbourne, Australia (HA); the Faculty of Health, School of Medicine, Deakin University, Waurn Ponds, Victoria, Australia (HA).

Received February 7, 2017. Final Revisions Submitted April 5, 2017. Date of ACCEPTANCE MAY 15, 2017.

Correspondence to: Sheng-Fei Oon, Cancer Imaging, Peter MacCallum Cancer Centre, Grattan Street, VIC 3000, Melbourne, Australia. Email: sheng.oon@ petermac.org. 

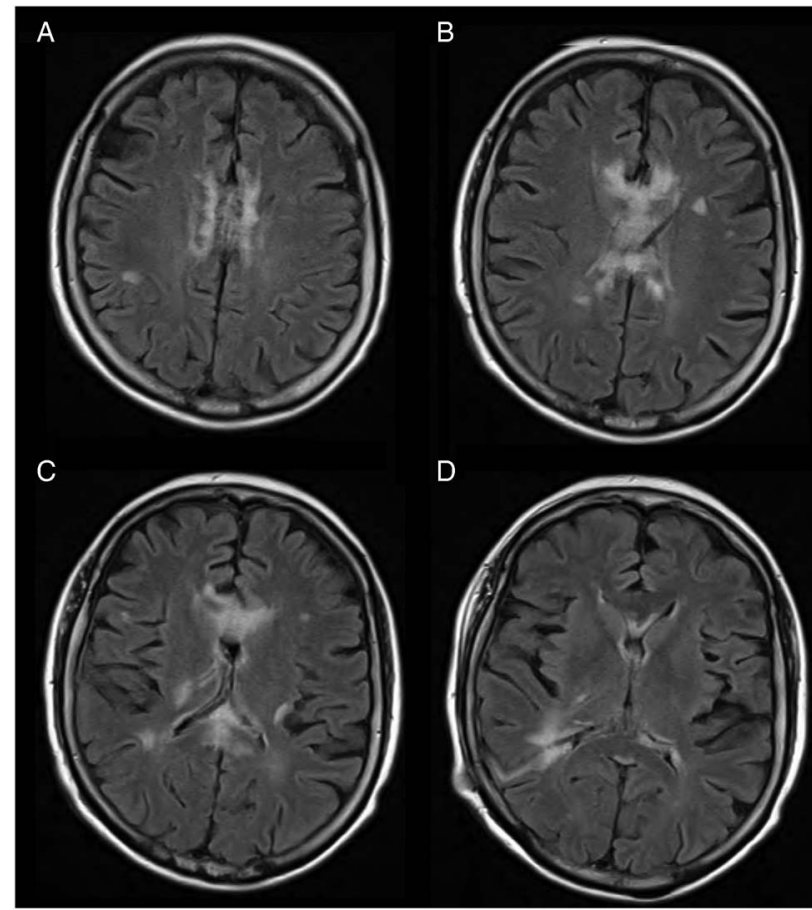

D

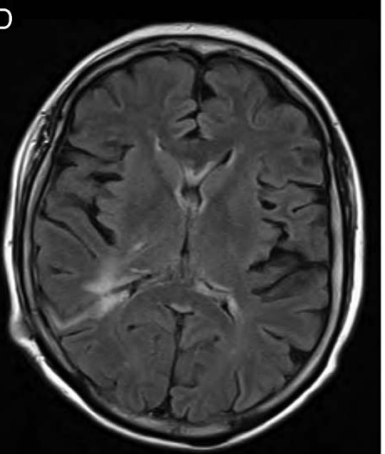

Figure 2: The lateral ventricles are collapsed following decompression by a ventriculoperitoneal shunt. There is extensive confluent FLAIR hyperintensity in the corpus callosum and pericallosal region not seen on the pre-decompression image $(C)$.

reversible changes. ${ }^{1}$ In another case series, obstructive hydrocephalus, usually from aqueductal stenosis, was the culprit mechanism for subsequent CCIS, as in the present case. CCIS was

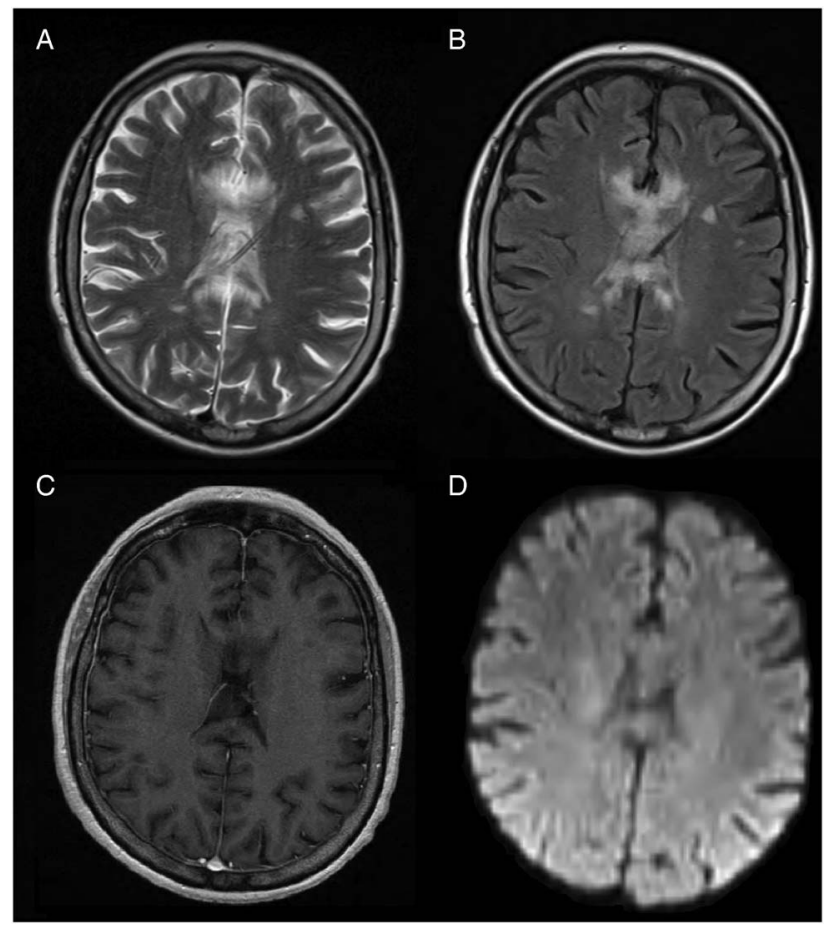

Figure 3: Axial sequences acquired through the corpus callosum demonstrate callosal and pericallosal T2 and FLAIR hyperintensity (A, $B)$ with no post-gadolinium enhancement $(C)$ or abnormal diffusion restriction $(D)$.

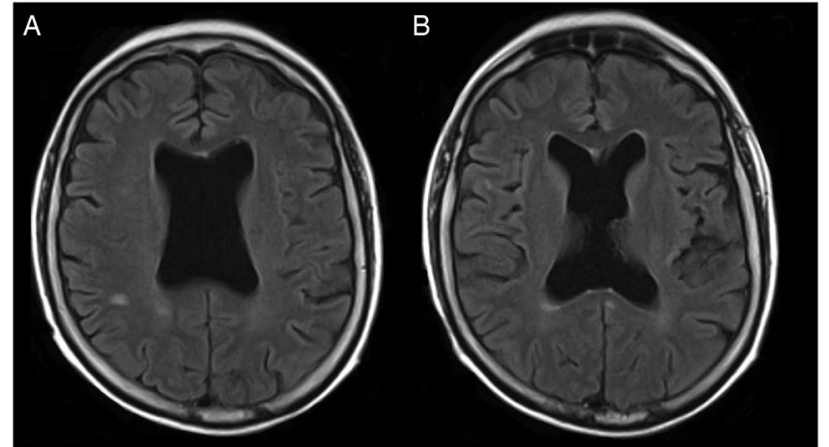

Figure 4: Axial T2 FLAIR sequences performed four weeks later demonstrating resolution of the callosal and pericallosal abnormalities. Note that there is recurrence of hydrocephalus, which was due to dislodgement of the ventriculoperitoneal shunt catheter, which required revision.

not associated with cases of communicating hydrocephalus. ${ }^{3}$ No long-term neurological sequelae from the callosal damage itself have been documented. ${ }^{3}$

When presented with a $\mathrm{T} 2$ signal abnormality of the corpus callosum, the lack of contrast enhancement and diffusion restriction, along with evidence of ventricular decompression in some cases, provide important clues to differentiate CCIS from more sinister causes of transcallosal signal abnormality-such as glioblastoma, lymphoma, and tumefactive demyelination.

\section{Statement of Authorship}

Sheng-Fei Oon was the principal author. Hong Kuan Kok was responsible for the text and editing. Christen D. Barras was responsible for the text and editing. Seamus Looby was the clinical director. Paul Brennan was a contributing author. Hamed Asadi was the lead clinician.

\section{Disclosures}

None of the authors have any financial or intellectual conflicts of interests to declare.

\section{REFERENCES}

1. Numaguchi Y, Kristt DA, Joy C, Robinson WL. Scalloping deformity of the corpus callosum following ventricular shunting. AJNR Am J Neuroradiol. 1993;14(2):355-62.

2. Spreer J, Ernestus RI, Lanfermann H, Lackner K. Lesions of the corpus callosum in hydrocephalic patients with ventricular drainage: a CT-study. Acta Neurochir (Wien). 1996;138(2): 174-8.

3. Lane JI, Luetmer PH, Atkinson JL. Corpus callosal signal changes in patients with obstructive hydrocephalus after ventriculoperitoneal shunting. AJNR Am J Neuroradiol. 2001;22(1):158-62.

4. Suh DY, Gaskill-Shipley M, Nemann MW, Tureen RG, Warnick RE. Corpus callosal changes associated with hydrocephalus: a report of two cases. Neurosurgery. 1997;41(2):488-93.

5. Bourekas EC, Varakis K, Bruns D. et al. Lesions of the corpus callosum: MR imaging and differential considerations in adults and children. Am J Roentgenol. 2002;179(1):251-7.

6. Lövblad KO, Anzalone N, Dörfler A, et al. MR imaging in multiple sclerosis: review and recommendations for current practice. AJNR Am J Neuroradiol. 2010;31(6):983-9. 